\title{
Localized conjunctival amyloidosis due to familial Mediterranean fever
}

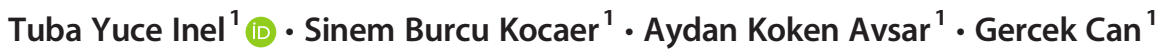

Received: 15 September 2020 / Revised: 15 October 2020 / Accepted: 18 October 2020 / Published online: 27 October 2020

(C) International League of Associations for Rheumatology (ILAR) 2020

\section{Presentation}

A 32-year-old female presented with left-sided orbital mass, ptosis, and subconjunctival bleeding (Fig. 1a). Increased thickness of the left upper eyelid was detected in cranial magnetic resonance imaging (MRI) (Fig. 1b). The workup included a complete blood count, angiotensin-converting enzyme, calcium, PTH, thyroid function tests, and IgG4 levels that were within normal limits. Rheumatoid factor, antinuclear antibody, and antineutrophilic cytoplasmic antibody tests were negative. Hematoxylin-eosin staining of the biopsy specimen demonstrated homogeneous, amorphous eosinophilic material in the conjunctival stroma. Under polarized light, the Congo red-stained material revealed a green birefringence characteristic of amyloid deposition. Negative IgG4 staining was detected. There was no organomegaly on physical examination. She had no proteinuria, and needle biopsy of abdominal subcutaneous fat was negative for amyloidosis. Cardiac MRI and bone marrow biopsy were normal. When the detailed history was obtained from the patient, it was learned that she had been experiencing abdominal pain, fever, and occasional pleuritic pain attacks since the age of 18 . The patient with heterozygous R202Q polymorphism and good clinical response to colchicine was diagnosed with familial Mediterranean fever (FMF). We present a case of FMF presenting with isolated eyelid and conjunctival amyloidosis.

\section{Discussion}

The presence of systemic involvement should be investigated in all patients and the patients should be questioned in detail regarding the etiology. Although cases of uveitis, scleritis, episcleritis, conjunctivitis, retrobulbar optic neuritis, and ophthalmoparesis secondary to FMF have been reported [1], presentation with conjunctival amyloidosis without systemic involvement is extremely rare.
Tuba Yuce Inel

dr.tubayuce@yahoo.com

1 Department of Rheumatology, Romatoloji BD, Ic Hastaliklari ABD, Tip Fakultesi, Dokuz Eylul University, Faculty of Medicine, Balcova, PK 35340 Izmir, Turkey 
Fig. 1 a Subconjunctival amyloid vegetations. b Increased thickness of the left upper eyelid

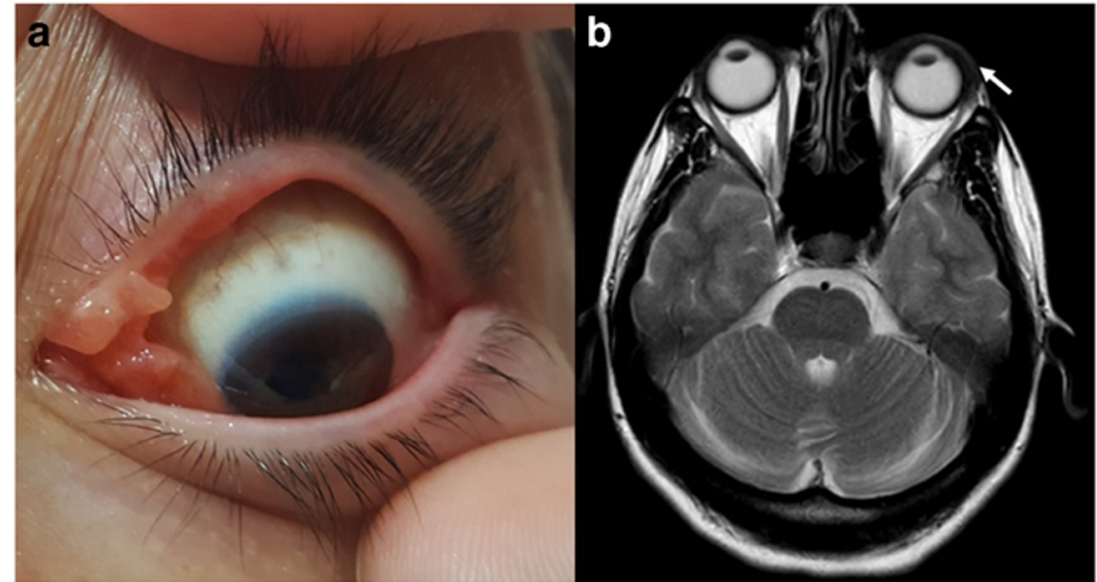

\section{Compliance with ethical standards}

Disclosures None.

\section{Reference}

1. Bascherini V, Granato C, Lopalco G, Emmi G, Vannozzi L, Bacherini D, Franceschini R, Iannone F, Salerni A, Molinaro F,
Messina M, Frediani B, Selmi C, Rigante D, Cantarini L (2015) The protean ocular involvement in monogenic autoinflammatory diseases: state of the art. Clin Rheumatol 34:1171-1180. https:// doi.org/10.1007/s10067-015-2920-3

Publisher's note Springer Nature remains neutral with regard to jurisdictional claims in published maps and institutional affiliations. 